\section{(4) \\ Check for \\ updates}

\section{Original Article}

\title{
Dual antiplatelet treatment associated with reduced risk of symptomatic vasospasm and delayed cerebral ischemia in aneurysmal subarachnoid hemorrhage
}

\author{
Gyeong-Ung Sun, Eunsung Park, Dae-Won Kim, Sung-Don Kang \\ Department of Neurosurgery, Institute of Wonkwang Medical Science, School of Medicine, \\ Wonkwang University, Iksan, Korea
}

\section{$\mathrm{J}$ Cerebrovasc Endovasc Neurosurg. 2020 September;22(3):134-140 Received: 24 May 2020 Revised: 7 July 2020 Accepted: 8 July 2020}

\section{Correspondence to Dae-Won Kim}

Department of Neurosurgery, School of Medicine, Wonkwang University, 895 Muwang-ro, Iksan 54538, Korea

Tel +82-63-859-1466

Fax +82-63-852-2606

E-mail kimdw@wku.ac.kr ORCID http://orcid.org/0000-0003-2151-2841
This is an Open Access article distributed under the terms of the Creative Commons Attribution Non-Commercial License (http://creativecommons.org/licenses/ by-nc/3.0/) which permits unrestricted noncommercial use, distribution, and reproduction in any medium, provided the original work is properly cited.
Objective: Cerebral vasospasm and delayed cerebral ischemia (DCl) are considered complications after aneurysmal subarachnoid hemorrhage (aSAH). Several hypotheses involving platelet activation have been asserted in the pathophysiology of cerebral vasospasm and $\mathrm{DCl}$. This study aimed to investigate the effect of dual antiplatelet treatment (DAPT) on symptomatic vasospasm and DCI in patients with aSAH.

Methods: A retrospective study was conducted on patients with aSAH from 2009 to 2018. The patients are divided into 2 groups according to the treatment method such as simple or balloon-assisted coil embolization group (SB coiling), and stentassisted coil embolization group. Patients treated by SB coiling without DAPT were classified as the control group. Patients who required dual antiplatelet treatment due to stent-assisted coil embolization were classified as DAPT group. The incidence of symptomatic vasospasm and $\mathrm{DCl}$ was compared between the two groups.

Results: Of 743 patients with aSAH, 563 patients were treated with clipping, 115 patients treated with SB coiling, and 65 patients receive stent-assisted coiling. Among 115 patients underwent SB coiling, 14 patients were excluded by the exclusion criteria. Total number of control group (SB coiling) was 101, DAPT group (stentassisted coiling) was 65. Depending on whether or not taking DAPT, the incidence of symptomatic vasospasm was lower in the DAPT group $(p=0.010)$. DCl incidence was also lower in the DAPT group, which was statistically significant ( $p=0.029)$.

Conclusions: DAPT reduces the frequency of symptomatic vasospasm and $\mathrm{DCl}$ in patients with aSAH in our single-center study. To warranting this topic, further, larger prospective and randomized studies should be needed.

Keywords Subarachnoid hemorrhage, Cerebral vasospasm, Delayed cerebral ischemia, Antiplatelet drug 


\section{INTRODUCTION}

Aneurysmal subarachnoid hemorrhage (aSAH) accounts for 2-5\% of all stroke. ${ }^{7) 14)}$ This SAH causes several complications that lead to cognitive impairment and disabilities, significantly worsening their quality of life. Cerebral vasospasm and delayed cerebral ischemia (DCI) were identified as major complications of SAH, with approximately $17-40 \%$ of patients with SAH developing persistent neuropathy and dying. ${ }^{17)}$

Symptoms, however, do not occur in all cases. Clinical or symptomatic vasospasm is defined as a neurological deficit accompanied by angiographic vasospasm and develops in $20-40 \%$ of patients with SAH with cerebral aneurysm rupture. ${ }^{210)}$ Despite the extensive research effort for decades, there were no specific pharmacological treatments. DCI is defined as a delayed onset of neurologic deterioration which last for $>1$ hour and in addition to a newly confirmed cerebral infarction at imaging study, such as computed tomography (CT) or magnetic resonance imaging (MRI), and develops in approximately $30 \%$ of patients with aSAH. The mechanisms of DCI are still unclear, but several hypotheses are presented including vasospasm, early brain injury, microthrombi, cortical spreading depolarization, bloodbrain barrier disruption, inflammation and impaired cerebral autoregulation. ${ }^{1116)}$ These mechanisms are very similar to those of cerebral vasospasm.

Referred to these hypotheses, we suggested that, antiplatelet drug could reduce inflammation and microthrombi formation, and finally reduce the incidence of DCI. Generally, a dual platelet aggregation inhibitor (aspirin, clopidogrel) is commonly used in the insertion of stents and flow diverters during endovascular aneurysm treatment. The relationship between dual antiplatelet treatment (DAPT) and symptomatic vasospasm and DCI has not been widely studied, thus we investigated the effect of DAPT on symptomatic vasospasm and DCI.

\section{MATERIALS AND METHODS}

This study was approved by the local institutional review board. A retrospective single-center study was conducted on patients with aSAH treated at our center from January 2009 to December 2018. In patients with aSAH, CT, CT angiography (CTA) and MRI were essentially performed, and transfemoral cerebral angiography (TFCA) was also performed if necessary. Information about modified world federation of neurosurgical societies (WFNS) grade, whether antiplatelet was taken because of other disease, such as cerebral infarction or coronary arterial disease, modified fisher scale on admission brain CT was acquired. Also, we investigated whether or not external ventricular drainage (EVD) and ventriculoperitoneal shunt was done due to hydrocephalus and hemorrhagic complication caused by surgical treatment of hydrocephalus. The definition of modified WFNS grade, modified fisher scale are described in Table 1. For the treatment of patients with aSAH, clipping or coiling (simple or balloon-assisted [SB], stent-assisted coiling) was performed considering the patient's age and location and size of aneurysm. Patients who were treated with stent-assisted coil embolization and had to take dual antiplatelet drug were classified as the DAPT

Table 1. Definition of modified WNFS grade, modified fisher scale

\begin{tabular}{ccc}
\hline & Grade & Definition \\
\hline Grade 1 & GCS 15 \\
Grade 2 & GCS 14 \\
& Grade 3 & GCS 13 \\
& Grade 4 & GCS 7-12 \\
& Grade 5 & GCS 3-6 \\
\hline Grade 1 & Thin SAH $(<1 \mathrm{~mm})$ \\
& No IVH \\
Grade 2 & Thin SAH $(<1 \mathrm{~mm})$ \\
& IVH present \\
& Grade 3 & Thick SAH $(>1 \mathrm{~mm})$ \\
& No IVH \\
& Grade 4 & Thick SAH $(>1 \mathrm{~mm})$ \\
& IVH present
\end{tabular}

WFNS, world federation of neurosurgical societies; GCS, glascow coma scale; SAH, subarachnoid hemorrhage; IVH, intraventricular hemorrhage 
group. In DAPT group, aspirin $100 \mathrm{mg}$ and clopidogrel $75 \mathrm{mg}$ per day were given after aneurysmal treatment. Those who were trated with coil embolization without stent (SB coiling) were classified as the control group. In the cases of neurologic deterioration. vascular studies such as CTA, MRA and TFCA were performed and if vasospasm was suspected, intra-arterial or balloon angioplasty was performed by two experts: one vascular neurosurgeon and one vascular neuroradiologist.

\section{Inclusion/Exclusion criteria}

Patients who treated with aSAH were included in this study. In order to eliminate bias due to differences in treatment modality, only patients with endovascular treatment were selected except surgical clipping. Exclusion criterias were followed: 1) patients with SAH caused by other vascular lesions such as arteriovascular malformation rupture or without prominent aneurysm. 2) control group patients taken antiplatelet drugs due to protrusion of coil mass into parent artery. 3) patients who had previously taken antiplatelet drugs due to other diseasse in control group.

\section{Definition of symptomatic vasospasm / DCI}

Symptomatic vasospasm is defined as neurological deterioration, such as decrease in level of consciousness or motor grade, following narrowing of the cerebral artery. DCI is defined as presence of diffusion restriction in diffusion weighted image of MRI or low density lesions newly developed on CT scan.

\section{Statistical analysis}

The differences in the incidence of symptomatic vasospasm and DCI between the control and DAPT groups and between two treatment modality (SB coiling vs. stent-assisted coiling) were compared. Categorical variables are presented as number (\%) and compared using Fisher's exact test or chi-square test. Statistical analyses were performed using IBM SPSS Statistics version 26.0 (IBM Corp., Armonk, NY, USA).

\section{RESULTS}

A total of 1,397 patients were treated for cerebral aneurysm, and 743 patients (53.2\%) were treated for ruptured cerebral aneurysms. There were 563 patients (75.8\%) who were treated with clipping, and 180 (24.2\%) who were treated with endovascular treatment. SB coiling was performed in 115 patients (63.9\%), while stent-assisted coiling was performed in 65 patients (36.1\%). Of 115 SB coiling patients, 14 patients were excluded from this study because they had previously taken antiplatelet drugs. Finally, 101 (60.8\%) patients in control group and 65 (39.2\%) paitnets in DAPT group were selected (Fig. 1). A total of 66 patients (8.9\%) had symptomatic vasospasm, while 56 patients $(7.5 \%)$ had DCI. The average period to the development of symptomatic vasospasm was 7.6 days (range, 2-17 days).

Based on the location of the ruptured cerebral aneurysm, the most frequent site was the anterior communicating artery (A-ComA), followed by the posterior communicating artery (P-ComA). Demographics of patient treated with endovascular method is presented at Table 2. The demographics of two groups were similar in age, sex, location of aneurysm, modified fisher scale, modified WFNS grade, hydrocephalus ( $p>0.05)$.

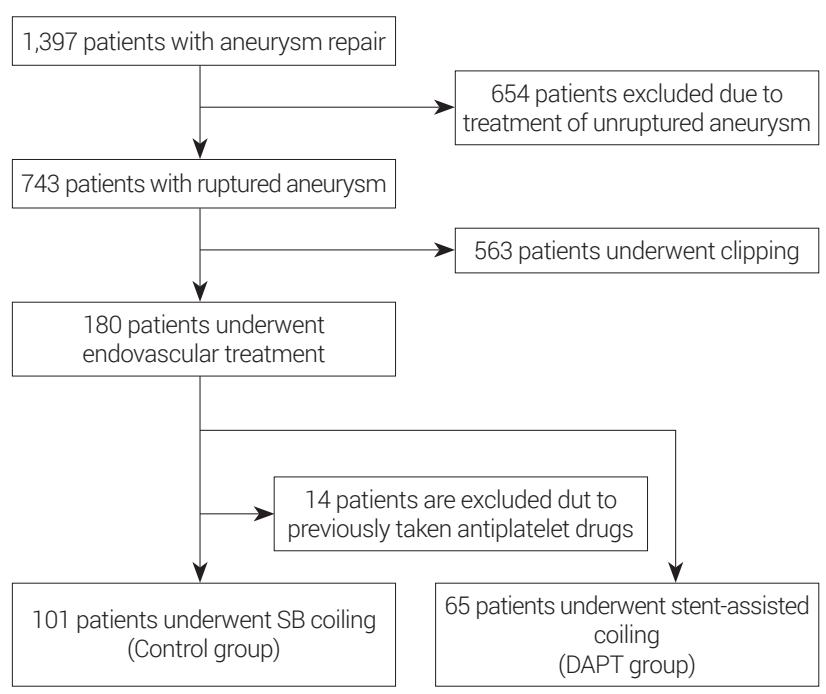

Fig. 1. Inclusion and exclusion criteria and classification data of patients in this study. SB, simple or balloon-assisted; DAPT, dual antiplatelet treatment. 
Table 2. Demographics of patients treated with endovascular methods

\begin{tabular}{|c|c|c|c|}
\hline Parameter & Control group (SB coiling) & DAPT group (Stent-assisted coiling) & $p$ value \\
\hline No. of patients & $101(60.8)$ & $65(39.2)$ & \\
\hline Mean age (yrs) & 63.4 & 65.1 & 0.472 \\
\hline Male sex & $26(25.7)$ & $14(21.5)$ & 0.539 \\
\hline Aneurysm location & & & 0.536 \\
\hline A-ComA & $22(21.8)$ & $4(6.2)$ & \\
\hline MCA & $8(7.9)$ & $2(3.1)$ & \\
\hline P-ComA & $19(18.8)$ & $24(36.9)$ & \\
\hline ICA & $6(5.9)$ & $11(16.9)$ & \\
\hline Distal ACA & $1(1.0)$ & $1(1.5)$ & \\
\hline Post. Circulation & $45(44.6)$ & $23(35.4)$ & \\
\hline Modified fisher scale & & & 0.629 \\
\hline Grade 1 & $4(4.0)$ & $3(4.6)$ & \\
\hline Grade 2 & $19(18.8)$ & $4(6.2)$ & \\
\hline Grade 3 & $22(21.8)$ & $25(38.4)$ & \\
\hline Grade 4 & $56(55.4)$ & $33(50.8)$ & \\
\hline Modified WFNS grade & & & 0.750 \\
\hline Grade 1 & $31(30.7)$ & $24(36.9)$ & \\
\hline Grade 2 & $23(22.8)$ & $11(16.9)$ & \\
\hline Grade 3 & $12(11.9)$ & $7(10.8)$ & \\
\hline Grade 4 & $16(15.8)$ & $12(18.5)$ & \\
\hline Grade 5 & $19(18.8)$ & $11(16.9)$ & \\
\hline Hydrocephalus & $41(40.6)$ & $27(41.5)$ & 0.905 \\
\hline EVD & $41(40.6)$ & $27(41.5)$ & 0.905 \\
\hline EVD hemorrhagic complication & $4(4.0)$ & $3(4.6)$ & 0.839 \\
\hline Ventriculoperitoneal shunt & $12(11.9)$ & $9(13.8)$ & 0.712 \\
\hline Ventriculoperitoneal shunt hemorrhagic complication & $3(3.0)$ & $2(3.1)$ & 0.969 \\
\hline
\end{tabular}

Values are presented as number (\%) unless otherwise indicated.

$\mathrm{SB}$, simple or balloon-assisted; DAPT, dual antiplatelet treatment; A-ComA, anterior communicating artery; MCA, middle cerebral artery; P-ComA, posterior communicating artery; ICA, internal carotid artery; ACA, anterior cerebral artery; WFNS, world federation of neurosurgical societies; EVD, external ventricular drainage

\section{Difference in vasospasm rate according to treatment modality of ruptured aneurysm}

We made a comparison between DAPT group and control group in accordance with the frequency of vasospasm. Among 101 patients in control group, 13 patients (12.9\%) have vasospasm. In contrast, among 65 patients in DAPT group, only 1 patient (1.5\%) suffered from vasospasm (Table 3 ). There was statistically significant difference between the two groups $(p=0.010)$.

\section{Difference in DCI incidence rate according to treatment modality of ruptured aneurysm}

Of a total of 738 patients with aSAH, 56 patients (7.5\%)
Table 3. Frequency of symptomatic vasospasm by treatment modality

\begin{tabular}{lccc}
\hline Parameter & $\begin{array}{c}\text { Control group } \\
\text { (SB coiling) }\end{array}$ & $\begin{array}{c}\text { DAPT group } \\
\text { (Stent-assisted coiling) }\end{array}$ & $\boldsymbol{p}$ value \\
\hline aSAH & 101 & 65 & \\
Vasospasm & 13 & 1 & 0.010 \\
$\begin{array}{l}\text { Frequency of } \\
\text { vasospasm (\%) }\end{array}$ & 12.9 & 1.5 &
\end{tabular}

SB, simple or balloon-assisted; DAPT, dual antiplatelet treatment; aSAH, aneurysmal subarachnoid hemorrhage

developed DCI. When compaed in the endovascular group that is the focus of this study, $11(10.9 \%)$ of the 101 patients in control group had DCI and 1 (1.5\%) of 65 patients in DAPT group had DCI (Table 4). Statistically 
Table 4. Frequency of DCI by treatment modality

\begin{tabular}{lccc}
\hline Parameter & $\begin{array}{c}\text { Control group } \\
\text { (SB coiling) }\end{array}$ & $\begin{array}{c}\text { DAPT group } \\
\text { (Stent-assisted coiling) }\end{array}$ & $\boldsymbol{p}$ value \\
\hline aSAH & 101 & 65 & \\
DCl & 11 & 1 & 0.029 \\
$\begin{array}{l}\text { Frequency of DCl } \\
(\%)\end{array}$ & 10.9 & 1.5 & \\
\hline
\end{tabular}

DCl, delayed cerebral ischemia; SB, simple or balloon-assisted; DAPT, dual antiplatelet treatment; aSAH, aneurysmal subarachnoid hemorrhage

significant difference ( $p=0.029)$ was observed between control and DAPT group.

\section{Difference in hemorrhagic complication depending on whether or not taking DAPT}

In control group, EVD related hemorrhagic complication occurred in 4 patients (4.0\%) and ventriculoperitoneal shunt related hemorrhagic complication occurred in 3 patients (3.0\%). In DAPT group, 3 patients (4.6\%) had EVD related hemorrhagic complication and 2 patients (3.1\%) had ventriculoperitoneal shunt related hemorrhagic complication. There was no statistical significance between two groups in regarding to EVD related hemorrhagic complication $(p=0.839)$ and ventriculoperitoneal shunt related hemorrhagic complication $(p=0.969)$.

\section{DISCUSSION}

Although there is no drug that has been clearly proven to prevent cerebral vasospasm and DCI, nimodipine, dihydropyridine-type calcium channel blocker (CCBs) have been sustained and used because they can potentially prevent cerebral vasospasm but not obviously reduce the incidence of DCI. ${ }^{9)}$ In the pathogenesis of cerebral vasospasm, smooth muscle contraction is an essential structural change. In early phase of cerebral vasospasm, smooth muscle contraction requires adenosine triphosphate (ATP) and calcium. Although myofilament activation depends on calcium and high-energy phosphate, chronid vasospasm does not. Unlike early phase, late phase of cerebral vasospasm needs contractile protein (protein kinase C, Rho kinase, etc.) instead of ATP and calcium. This second phase cerebral vasospasm is less reversible with pharmacologic vasodilator (CCBs) treatment. $^{5)}$

Studies have been conducted to find the relationship between antiplatelet drugs and prevention of vasospasm. There are some studies in which cilostazol, antiplatelet agent, can prevent cerebral vasospasm. Yoshimoto et al. ${ }^{18)}$ reported that vasospasm was less and statistically meaningful in the patient group who took cilostazol from first day of surgery than those who did not. Also, Senbokuya et al. ${ }^{12)}$ showed that cilostazol could prevent vasospasm. But, few studies are reported about the relationship between DAPT and vasospasm.

There are several hypotheses on the mechanism of cerebral vasospasm and DCI. Among them, we focused on the theory about inflammation and microthrombi, which are the basis for DAPT in this study. Inflammation is triggered by leakage of blood that occurs during aneurysmal rupture, resulting in increased secretion of leukotrienes and cytokines. This causes the endothelium to secrete endothelin-1 and change it to increase the use of NO, triggering vasospasm by the same mechanism noted earlier. ${ }^{4) 13}$ Moreover, the production of the pro-inflammatory marker "NF- $\kappa \mathrm{B}$ " by blood in the subarachnoid space increases, which increases the synthesis of cytokine and adhesion molecule. ${ }^{8)}$ Cytokine affects the maintenance of vasoconstriction, keeping the impact stronger and making vasospasm last longer. ${ }^{6}$ Although a clear mechanism has not found about microthrombi, the procoagulant state activated by increased expression of pro-inflammatory markers is influential. This activates the aggregation of leukocytes and platelets, resulting in formation of microthrombi. ${ }^{15)}$ DAPT can be used together to inhibit the activation and aggregation of platelets and to suppress the formation of microthrombi. Based on this mechanisms, we suggested that inflammatory pathway activation can be suppressed too.

Cagnazzo et al. ${ }^{1}$ reported that the difference in the incidence of DCI due to the use of antiplatelet drugs was not statistically significant. However, in the endovascular treated group with antiplatelet therapy, DCI tends to 
have a lower incidence compared to that in the group without antiplatelet treatment (157/778 vs. $413 / 1,410$, odds ratio $[\mathrm{OR}]=0.552 ; 95 \%$ confidence interval $[\mathrm{CI}]$, 0.273-1.115, $p=0.06$ ). Moreover, when antiplatelet therapy was applied for more than 2 weeks, the incidence of DCI tended to be lower (63/438 vs. $96 / 353$, $\mathrm{OR}=0.379 ; 95 \% \mathrm{CI}, 0.12-1.2 ; p=0.06$ ). Additionally, Darkwah et al. ${ }^{3)}$ showed when aspirin was used after aSAH treatment, the incidence of DCI tended to be lower, which was also statistically significant $(p<0.001$, adjusted $\mathrm{OR}=0.41,95 \% \mathrm{CI} 0.24-0.65$ ).

\section{Incidence of vasospasm in the HIRA database}

For comparison with more patients, data from the Health Insurance Review and Assessment Service (HIRA) sample dataset (2012-2016) in the Korean nationwide database were also analyzed. Among total of 2,743 patients with aSAH, symptomatic vasospasm developed in 207 patients (7.5\%). When divided according to treatment modality, 1,421 patients (51.8\%) received clipping, 1,022 patients $(37.3 \%)$ received SB coiling, and 300 patients (10.9\%) received stent-assisted coiling. When compared with symptomatic vasospasm rate for each treatment method, 114 patients $(8.0 \%)$ were treated with clipping, 78 patients $(7.6 \%)$ were treated with SB coiling (control group), 15 patients (5.0\%) were treated with stent-assisted coiling (DAPT group). Although there was also no statistically significant difference in symptomatic vasospasm incidence between the control group and DAPT group $(p=0.07)$, but a tendency of low incidence of symptomatic vasospasm in the DAPT group was shown in this study.

\section{Limitations of this study}

This study has a few limitations: 1) This study was not randomized study. That is, use of DAPT was determined depending on the treatment method. 2) Although radial force of stent is weak, but possibility of the physical vasodilation effect caused by stent may affect vasospasm. However, since most stents are inserted into large vessels that are less prone to vasospasm such as ICA and posterior circulation, the effect of radial force caused by stent on vasospasm is thought to be negligible.

\section{CONCLUSIONS}

In this single-center study, DAPT after repair of aneurysm reduce the incidence of symptomatic vasospasm and DCI. To compensate for some limitations, further prospective and randomized studies should be concerned. For patients who have high risks for symptomatic vasospasm and DCI, it may be appropriate to consider the prophylactic use of DAPT after treatment of aneurysm.

\section{ACKNOWLEDGEMENTS}

This paper was supported by Wonkwang University in 2019.

\section{Disclosure}

The authors report no conflict of interest concerning the materials or methods used in this study or the findings specified in this paper.

\section{REFERENCES}

1. Cagnazzo F, Derraz I, Lefevre PH, Gascou G, Dargazanli C, Riquelme C, et al. Antiplatelet therapy in patients with aneurysmal SAH: impact on delayed cerebral ischemia and clinical outcome. A meta-analysis. AJNR Am J Neuroradiol. 2019 Jul;40(7):1201-6.

2. Castanares-Zapatero D, Hantson P. Pharmacological treatment of delayed cerebral ischemia and vasospasm in subarachnoid hemorrhage. Ann Intensive Care. 2011 May;1(1):12.

3. Darkwah Oppong M, Gembruch O, Pierscianek D, Köhrmann M, Kleinschnitz C, Deuschl C, et al. Post-treatment antiplatelet therapy reduces risk for delayed cerebral ischemia due to aneurysmal subarachnoid hemorrhage. Neurosurgery. 2019 Dec;85(6):827-33.

4. Dumont AS, Dumont RJ, Chow MM, Lin CL, Calisaneller T, Ley KF, et al. Cerebral vasospasm after subarachnoid hemorrhage: putative role of inflammation. Neurosurgery. 2003 Jul;53(1):123-33; discussion 133-5.

5. Findlay JM, Nisar J, Darsaut T. Cerebral vasospasm: a review. Can J Neurol Sci. 2016 Jan;43(1):15-32. 
6. Helmy A, De Simoni MG, Guilfoyle MR, Carpenter KL, Hutchinson PJ. Cytokines and innate inflammation in the pathogenesis of human traumatic brain injury. Prog Neurobiol. 2011 Nov;95(3):352-72.

7. Le Roux AA, Wallace MC. Outcome and cost of aneurysmal subarachnoid hemorrhage. Neurosurg Clin N Am. 2010 Apr;21(2):235-46.

8. Lucke-Wold BP, Logsdon AF, Manoranjan B, Turner RC, McConnell E, Vates GE, et al. Aneurysmal subarachnoid hemorrhage and neuroinflammation: a comprehensive review. Int J Mol Sci. 2016 Apr;17(4):497.

9. Mees SD, Rinkel GJ, Feigin VL, Algra A, van den Bergh WM, Vermeulen $M$, et al. Calcium antagonists for aneurysmal subarachnoid haemorrhage. Cochrane database of systematic reviews. Cochrane Database Syst Rev. 2007 Jul; 2007(3):CD000277.

10. Ohta H, Ito Z. Cerebral infarction due to vasospasm, revealed by computed tomography. Neurol Med Chir (Tokyo). 1981 Apr; 21(4):365-72.

11. Rowland MJ, Hadjipavlou G, Kelly M, Westbrook J, Pattinson KT. Delayed cerebral ischaemia after subarachnoid haemorrhage: looking beyond vasospasm. Br J Anaesth. 2012 Sep; 109(3):315-29.

12. Senbokuya N, Kinouchi H, Kanemaru K, Ohashi Y, Fukamachi
A, Yagi S, et al. Effects of cilostazol on cerebral vasospasm after aneurysmal subarachnoid hemorrhage: a multicenter prospective, randomized, open-label blinded end point trial. J Neurosurg. 2013 Jan;118(1):121-30.

13. Spallone A, Acqui M, Pastore FS, Guidetti B. Relationship between leukocytosis and ischemic complications following aneurysmal subarachnoid hemorrhage. Surg Neurol. 1987 Mar; 27(3):253-8

14. Suarez JI, Tarr RW, Selman WR. Aneurysmal subarachnoid hemorrhage. N Engl J Med. 2006 Jan;354(4):387-96.

15. Vergouwen MD, Vermeulen M, Coert BA, Stroes ES, Roos YB. Microthrombosis after aneurysmal subarachnoid hemorrhage: an additional explanation for delayed cerebral ischemia. J Cereb Blood Flow Metab. 2008 Nov;28(11):1761-70.

16. Wartenberg KE. Update on the management of subarachnoid hemorrhage. Future Neurol. 2013 Feb;8(2):205-24.

17. Wu CT, Wong CS, Yeh CC, Borel CO. Treatment of cerebral vasospasm after subarachnoid hemorrhage--a review. Acta Anaesthesiol Taiwan. 2004 Dec;42(4):215-22.

18. Yoshimoto T, Shirasaka T, Fujimoto S, Yoshidumi T, Yamauchi T, Tokuda K, et al. Cilostazol may prevent cerebral vasospasm following subarachnoid hemorrhage. Neurol Med Chir (Tokyo). 2009 Jun;49(6):235-40; discussion 240-1. 